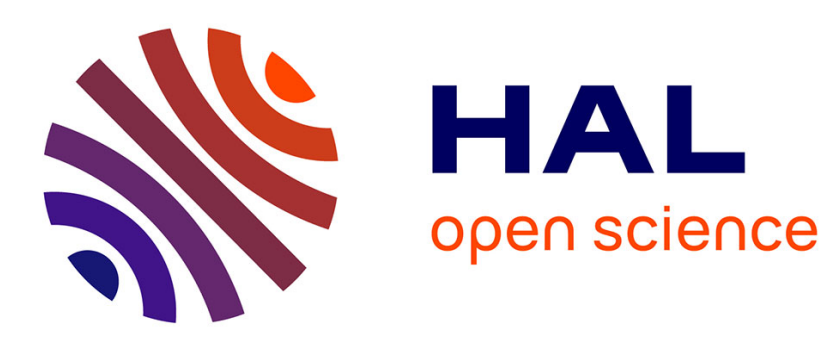

\title{
Hybrid Finite Element Method and Variational Theory of Complex Rays for Helmholtz Problems
}

\author{
Hao Li, Pierre Ladevèze, Hervé Riou
}

\section{To cite this version:}

Hao Li, Pierre Ladevèze, Hervé Riou. Hybrid Finite Element Method and Variational Theory of Complex Rays for Helmholtz Problems. Journal of Computational Acoustics, 2016, 24 (4), pp.1650015. 10.1142/S0218396X16500156 . hal-01647653

\section{HAL Id: hal-01647653 https://hal.science/hal-01647653}

Submitted on 21 Aug 2019

HAL is a multi-disciplinary open access archive for the deposit and dissemination of scientific research documents, whether they are published or not. The documents may come from teaching and research institutions in France or abroad, or from public or private research centers.
L'archive ouverte pluridisciplinaire HAL, est destinée au dépôt et à la diffusion de documents scientifiques de niveau recherche, publiés ou non, émanant des établissements d'enseignement et de recherche français ou étrangers, des laboratoires publics ou privés. 


\title{
Hybrid Finite Element Method and Variational Theory of Complex Rays for Helmholtz Problems
}

\author{
Hao $\mathrm{Li}^{*}$, Pierre Ladevèze ${ }^{\dagger}$ and Hervé Riou ${ }^{\ddagger}$ \\ LMT, ENS Cachan, CNRS, Université Paris-Saclay \\ F-94235 Cachan, France \\ *hao-li@lmt.ens-cachan.fr \\ ${ }^{\dagger}$ pierre.ladeveze@lmt.ens-cachan.fr \\ †herve.riou@lmt.ens-cachan.fr
}

\begin{abstract}
In this paper, we consider the Weak Trefftz Discontinuous Galerkin (WTDG) method, which enables one to use at the same time the Finite Element Method (FEM) or Variational Theory of Complex Rays (VTCR) discretizations (polynoms and waves), for vibration problems. It has already been developed such that the FEM and the VTCR can be used in different adjacent subdomains in the same problem. Here, it is revisited and extended in order to allow one to use the two discretizations in the same subdomain, at the same time. Numerical examples illustrate the performances of such an approach.
\end{abstract}

Keywords: Hybrid methods; Trefftz methods; discontinuous Galerkin methods; VTCR; FEM.

\section{Introduction}

Today, the numerical simulation has become indispensable to analyze and optimize the problems in every part of engineering processes. Based on the piecewise, continuous polynomial approximation, the standard Galerkin Finite Element Method (FEM) ${ }^{1}$ is a wellestablished method which is commonly used for the analysis of time-harmonic dynamic problems. However, it suffers from the pollution effect. ${ }^{2}$ As a consequence, when the wave number is increased, the meshes need drastically to be refined. This leads to inefficient calculations for problems when the medium and the high frequencies are considered. As the FEM is based on polynomial approximations, the problem cannot fundamentally be solved.

Other approaches have been developed in order to alleviate this problem, namely the Trefftz approaches. ${ }^{3}$ They are based on the use of exact approximations of the governing equation. Such methods are, for example, the partition of unity method, ${ }^{4}$ the ultra weak variational method, ${ }^{5,6}$ the least square method, ${ }^{7,8}$ the plane wave discontinuous Galerkin methods,${ }^{9}$ the method of fundamental solutions ${ }^{10,11}$ the discontinuous enrichment method, ${ }^{12,13}$ the element free Galerkin method, ${ }^{14}$ the wave boundary element method $^{15,16}$ and the wave based method. ${ }^{17,18}$ The Variational Theory of Complex Rays (VTCR), first introduced in 
Ref. 19, belongs to this category of numerical strategies which uses waves in order to get some approximations for vibration problems. It has been developed for 3D plate assemblies in Ref. 20, for plates with heterogeneities in Ref. 21 and for shells in Ref. 22. Its extensions to acoustics problems can be seen in Refs. 23 and 24. In opposition to FEM, the VTCR has good performances for medium frequency applications, but is less efficient for very low frequency problems.

Recently, a new approach called the Weak Trefftz Discontinuous Galerkin (WTDG) method has been introduced. ${ }^{25}$ It differs from the pure Trefftz methods, because the necessity to use exact solution of the governing equations can be weaken. Then it allows one to couple the FEM (based on polynoms) and the VTCR (based on waves) approximations at the same time. In the illustrations proposed Ref. 25, the polynoms and the waves approximations where used in different adjacent subdomains of a problem. Here, we propose to mix the approximations in the same subdomains, at the same time. This allow us to give to the strategy a strong flexibility for engineering problems. Through this paper, it will be shown that such a mix approach (which uses at the same time the polynomial and the wave descriptions) presents better performances than a pure FEM approach (which uses only a polynomial description) or a pure VTCR approach (which uses only a wave description).

This work is presented as follows: in Sec. 2 we present the reference problem and develop some fundamental properties of WTDG. In Sec. 3, we propose some numerical examples to illustrate the strategy. Conclusions are drawn in Sec. 4.

\section{The Weak Trefftz Discontinuous Galerkin Method}

\subsection{The reference problem}

The reference problem to solve is illustrated in the context of Helmholtz problem, which can be applied into acoustic vibration problems. Let us consider the domain $\Omega$ with the boundaries $\partial \Omega=\partial_{1} \Omega \cup \partial_{2} \Omega$, where $\partial_{1} \Omega$ corresponds to the boundary where Dirichlet conditions are prescribed, and $\partial_{2} \Omega$ corresponds to the boundary where Neumann conditions are prescribed (see Fig. 1). The following problem is considered: find $u \in H^{1}(\Omega)$
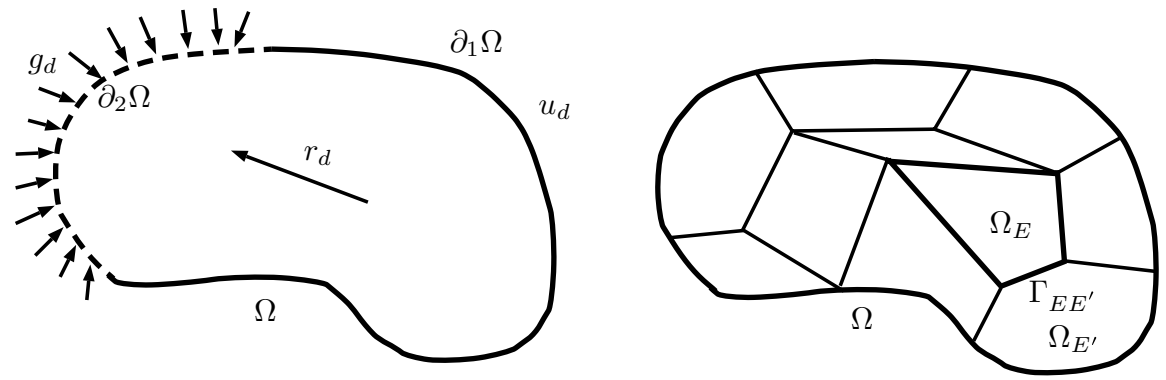

Fig. 1. Left: Definition of the computational domain $\Omega$. Right: Definition of the subdomains of $\Omega$. 
such that

$$
\begin{aligned}
(1+i \zeta) \Delta u+k^{2} u+r_{d} & =0 & & \text { over } \Omega, \\
u & =u_{d} & & \text { over } \partial_{1} \Omega, \\
(1+i \zeta) \partial_{n} u & =g_{d} & & \text { over } \partial_{2} \Omega,
\end{aligned}
$$

where $\partial_{n} u=\operatorname{grad} u \cdot \mathbf{n}$ and $\mathbf{n}$ is the outward normal. $u$ is the physical variable studied such as the pressure in acoustics. $\zeta$ is the damping coefficient, which is positive or equals to zero. $k$ is the wave number, which is a real, positive and constant value. $u_{d}$ and $g_{d}$ are the prescribed Dirichlet and Neumann data. The first equation of (1) is the standard Helmholtz equation. The second and third equations correspond to the boundary conditions.

\subsection{The WTDG variational formulation of the reference problem}

In order to get an equivalent variational formulation of (1), the domain is divided into subdomains $\Omega_{E}$ with $E \in \mathbf{E}$ (see Fig. 1). $\Gamma_{E E^{\prime}}$ denotes the interface between two subdomains $E$ and $E^{\prime} . \Gamma_{E E}$ denotes the interface between subdomain $\Omega_{E}$ and boundary $\partial \Omega$. The WTDG method consists in using the working space $\mathcal{U} \subset H^{1}(\Omega)$ :

$$
\begin{aligned}
\mathcal{U} & =\left\{u \mid u_{\mid \Omega_{E}} \in \mathcal{U}_{E}\right\}, \\
\mathcal{U}_{E} & =\left\{u_{E} \mid u_{E} \in \mathcal{V}_{E} \subset H^{1}\left(\Omega_{E}\right)\right\} .
\end{aligned}
$$

The vector spaces associated with $\mathcal{U}$ and $\mathcal{U}_{E}$ where $r_{d}=0$ are denoted by $\mathcal{U}_{0}$ and $\mathcal{U}_{E, 0}$.

Denoting

$$
\begin{aligned}
\{u\}_{E E^{\prime}} & =\left(u_{E}+u_{E^{\prime}}\right)_{\mid \Gamma_{E E^{\prime}}}, \\
{[u]_{E E^{\prime}} } & =\left(u_{E}-u_{E^{\prime}}\right)_{\mid \Gamma_{E E^{\prime}}}, \\
\mathbf{q}_{\mathbf{u}} & =(1+i \zeta) \operatorname{grad} u
\end{aligned}
$$

the WTDG formulation can be written ${ }^{25}$ : find $u \in \mathcal{U}$ such that

$$
\begin{aligned}
\operatorname{Re}(-i k & \left(\sum_{E, E^{\prime} \in \mathbf{E}} \int_{\Gamma_{E E^{\prime}}}\left(\frac{1}{2}\left\{\mathbf{q}_{\mathbf{u}} \cdot \mathbf{n}\right\}_{E E^{\prime}}\{\tilde{v}\}_{E E^{\prime}}-\frac{1}{2}\left[\tilde{\mathbf{q}}_{\mathbf{v}}\right]_{E E^{\prime}}[u]_{E E^{\prime}}\right) d S\right. \\
& -\sum_{E \in \mathbf{E}} \int_{\Gamma_{E E} \cap \partial_{1} \Omega} \tilde{\mathbf{q}}_{\mathbf{v}} \cdot \mathbf{n}\left(u-u_{d}\right) d S+\sum_{E \in \mathbf{E}} \int_{\Gamma_{E E} \cap \partial_{1} \Omega} \alpha \cdot i \cdot \tilde{v}\left(u-u_{d}\right) d S \\
& +\sum_{E \in \mathbf{E}} \int_{\Gamma_{E E} \cap \partial_{2} \Omega}\left(\mathbf{q}_{\mathbf{u}} \cdot \mathbf{n}-g_{d}\right) \tilde{v} d S \\
& \left.\left.-\sum_{E \in \mathbf{E}} \int_{\Omega_{E}}\left(\operatorname{div} \mathbf{q}_{\mathbf{u}}+k^{2} u+r_{d}\right) \tilde{v} d \Omega\right)\right)=0 \forall v \in \mathcal{U}_{0},
\end{aligned}
$$

where $\alpha$ is a parameter strictly positive to enforce the boundary Dirichlet condition. 
As one can see, there is no a priori constraint on the choice of the spaces $\mathcal{U}$ and $\mathcal{U}_{0}$. As a consequence, one can select polynoms, like in FEM, or waves, like in VTCR, or even both. This gives to the strategy a very strong flexibility.

\subsection{Properties of the WTDG formulation}

Let us note that (4) can be written as: find $u \in \mathcal{U}$ such that

$$
b(u, v)=l(v), \quad \forall v \in \mathcal{U}_{0},
$$

where $b$ and $l$, are $u$-linear in $\mathcal{U}$ and $v$-(anti)linear in $\mathcal{U}_{0} . b$ is such that $b(u, u)$ is real.

Property 1. For $u \in \mathcal{U}_{0}$, we have

$$
b(u, u)=\sum_{E \in \mathbf{E}} k \zeta \int_{\Omega_{E}} \operatorname{grad} \tilde{u} \cdot \operatorname{grad} u d \Omega+\sum_{E \in \mathbf{E}} \int_{\Gamma_{E E} \cap \partial_{1} \Omega} k \alpha u \tilde{u} d S \geq 0 .
$$

\section{Proof.}

$b(u, u)=\operatorname{Re}\left(-i k\left(\sum_{E \in \mathbf{E}} \int_{\partial \Omega_{E}}\left(\mathbf{q}_{\mathbf{u}} \cdot \mathbf{n}\right) \tilde{u} d S-\sum_{E \in \mathbf{E}} \int_{\Omega_{E}} \operatorname{div} \mathbf{q}_{\mathbf{u}} \tilde{u} d \Omega+\sum_{E \in \mathbf{E}} \int_{\Gamma_{E E} \cap \partial_{1} \Omega} \alpha i u \tilde{u} d S\right)\right)$.

Consequently,

$$
b(u, u)=\sum_{E \in \mathbf{E}} k \zeta \int_{\Omega_{E}} \operatorname{grad} \tilde{u} \cdot \operatorname{grad} u d \Omega+\sum_{E \in \mathbf{E}} \int_{\Gamma_{E E} \cap \partial_{1} \Omega} k \alpha u \tilde{u} d S .
$$

From Property 1 it can be deduced that if $b(u, u)=0$, then $u$ is equal to zero over $\partial \Omega_{E} \cap \partial_{1} \Omega$. It is a piecewise constant within subdomains $\Omega_{E}, E \in \mathbf{E}$ and is not equal to zero. To keep the uniqueness of the solution, condition $(\mathrm{P})$ is introduced to be satisfied by the shape functions which belong to $\mathcal{U}_{0}$.

Condition (P). Let $a_{E} \in \mathcal{U}_{E}$ be a piecewise constant function within subdomains $E \in \mathbf{E}$. $a_{E}$ satisfies condition $(\mathrm{P})$ if

$$
\left\{\forall v \in \mathcal{U}_{0}, \forall E \in \mathbf{E}, \operatorname{Re}\left(-i k\left(\sum_{E, E^{\prime} \in \mathbf{E}} \int_{\partial \Omega_{E}}\left(\mathbf{q}_{\mathbf{v}} \cdot \mathbf{n}\right) \tilde{a}_{E^{\prime}} d S\right)\right)=0\right\} \Rightarrow a_{E}= \pm a,
$$

where $E^{\prime}$ is a subdomain sharing a common boundary with $E$. And let us take the convention $a_{E^{\prime}}=-a_{E}$ over $\partial \Omega_{E} \cap \partial \Omega$.

Property 2. If $\mathcal{U}_{0}$ satisfies condition (P) and if $\zeta$ is positive, the WTDG formulation (4) has a unique solution. 
Proof. Existence of solution is evident. And suppose (4) has two solutions $u^{1}$ and $u^{2}$. $v=u^{1}-u^{2} \in \mathcal{U}_{0}$ and

$$
b(v, v)=\sum_{E \in \mathbf{E}} k \zeta \int_{\Omega_{E}} \operatorname{grad} \tilde{v} \cdot \operatorname{grad} v d \Omega+\sum_{E \in \mathbf{E}} \int_{\Gamma_{E E} \cap \partial_{1} \Omega} k \alpha v \tilde{v} d S=0 .
$$

It can be observed that $v_{E}=a_{E}$ with $E \in \mathbf{E}$, where $a_{E}$ is piecewise constant within the subdomains and $a_{E}=0$ in the subdomains sharing a common boundary with $\partial_{1} \Omega$. Back-substituting this result into (4), one also finds $b\left(v, v^{*}\right)=0 \forall v^{*} \in \mathcal{U}_{0}$, which leads to

$$
\forall v^{*} \in \mathcal{U}_{0}, \quad \operatorname{Re}\left(-i k\left(\sum_{E, E^{\prime} \in \mathbf{E}} \int_{\partial \Omega_{E}}\left(\mathbf{q}_{\mathbf{v}} \cdot \mathbf{n}\right) \tilde{a}_{E^{\prime}} d S\right)\right)=0 .
$$

Equation (11) corresponds to the condition $(\mathrm{P})$, where $E^{\prime}$ represents a subdomain sharing a common boundary with $E$, with the convention $a_{E^{\prime}}=-a_{E}$ over $\partial \Omega_{E} \cap \partial \Omega$. Consequently, $a_{E}= \pm a \forall E \in \mathbf{E}$. Moreover, given that $a_{E}=0$ over $\partial_{1} \Omega$, we have $a=0$.

Property 3. If $\mathcal{U}_{E}$ is the combination of solution spaces of FEM and VTCR, then the condition $(\mathrm{P})$ is satisfied, and

$$
\|u\|_{\mathcal{U}_{0}}^{2}=b(u, u)+\gamma^{2}(u)
$$

is a norm over $\mathcal{U}_{0}$, with the definition

$$
\overline{\mathcal{U}}_{E, 0}=\left\{u \mid u \in \mathcal{V}_{E}, u=\mathbf{C}_{\mathbf{E}} \cdot \mathbf{X}_{\mathbf{E}}\right\}
$$

where $\mathbf{C}_{\mathbf{E}}$ is constant over $\Omega_{E}$ and $\mathbf{X}_{E}$ is the position vector relative to the center of inertia of element $E . \overline{\mathcal{U}}_{0}$ denotes the associated space defined over $\Omega$ of $\overline{\mathcal{U}}_{E, 0}$. And for $u \in \mathcal{U}_{0}$ the definition of quantity $\gamma$ is introduced as

$$
\gamma(u)=\sup _{v \in \overline{\mathcal{U}}_{0}} b(u, v) /\left\|\mathbf{C}_{\mathbf{v}}\right\|_{L^{2}(\Omega)},
$$

where $\mathbf{C}_{\mathbf{v}}$ corresponds to the vector $\mathbf{C}_{\mathbf{E}}$ of $v$ according to (13).

Proof. To demonstrate that condition $(\mathrm{P})$ is satisfied, let us take $a_{E} \in \mathcal{U}_{E}, E \in \mathbf{E}$ a piecewise constant. Since $\mathcal{U}_{E}$ is the combination of FEM and VTCR, $u$ could be any linear combination of polynoms and wave functions. Therefore when $u=a_{E}$ a piecewise constant, $u$ could only be the polynomial function of order 0 . Let us note that $\forall E \in \mathbf{E} a_{E^{\prime}}=\beta_{E}$ $\left(\beta_{E}\right.$ constant over $\left.\Omega_{E}\right)$ for any subdomain $E^{\prime}$ sharing a common boundary with $E$. Let us introduce $z_{E}=\beta_{E}+a_{E} \cdot z_{E}$ is continuous because $z_{E \mid \Gamma_{E E^{\prime}}}=a_{E^{\prime}}+a_{E}=z_{E^{\prime} \mid \Gamma_{E E^{\prime}}}$. It follows that $z$ is constant over $\Omega$. Since $z$ is zero over $\partial \Omega, z=0$ over $\Omega$ and $\beta_{E}=-a_{E}$. Consequently, $a_{E}$ can be only the values of $+a$ or $-a, a$ being a constant over $\Omega$.

To demonstrate that $\|u\|_{\mathcal{U}_{0}}^{2}$ is a norm over $\mathcal{U}_{0}$, let us consider that $\|u\|_{\mathcal{U}_{0}}^{2}=b(u, u)+$ $\gamma^{2}(u)=0$. It follows that $b(u, u)=0$ and $\gamma(u)=0$. From (8) it can be obtained that 
$u_{\mid \Omega_{E}}=a_{E}$ is constant over $\Omega_{E}$ and that $u=0$ over $\partial_{1} \Omega$. Then $\gamma(u)$ is equal to

$$
\gamma(u)=\sup _{v \in \overline{\mathcal{U}}_{0}} \frac{1}{\left\|\mathbf{C}_{\mathbf{v}}\right\|_{L^{2}(\Omega)}} \operatorname{Re}\left(-i k\left(\sum_{E, E^{\prime} \in \mathbf{E}} \int_{\partial \Omega_{E}}\left(\mathbf{q}_{\mathbf{v}} \cdot \mathbf{n}\right) \tilde{a}_{E^{\prime}} d S\right)\right)=0 .
$$

Since condition $(\mathrm{P})$ is satisfied, it can be derived that $u_{E}= \pm a, a$ being a constant over $\Omega$. Finally from $u=0$ on $\partial_{1} \Omega$, one gets $u=0$ over $\Omega$.

\subsection{Implementation of the WTDG method}

As said before, we here seek the approximation solution as a sum of traditional FEM shape functions and traditional VTCR shape functions. As a consequence, we can write

$$
\begin{aligned}
\mathcal{U}=\left\{\begin{array}{l}
u \\
u
\end{array}\right. & L^{2}(\Omega): u(\mathbf{x})_{\mid \Omega_{j}}=\sum_{n_{j}=1}^{N_{j}} \lambda_{n_{j}} \varphi^{n_{j}}(\mathbf{x}) \\
& \left.+\sum_{m_{j}=1}^{M_{j}} A_{m_{j}} e^{i k \mathbf{d}_{m_{j}} \cdot \mathbf{x}}, \lambda_{n_{j}} \in \mathbb{C}, A_{m_{j}} \in \mathbb{C}, j=1, \ldots, n_{s}\right\},
\end{aligned}
$$

where $\varphi^{n}$ correspond to the standard, continuous piecewise polynomial FEM shape function, associated to the unknown degree of freedom $\lambda_{n}$, and $e^{i k \mathbf{d}_{m} \cdot \mathbf{x}}$ is the standard wave VTCR shape function, associated to the unknown degree of freedom $A_{m}\left(\mathbf{d}_{m}\right.$ is the unitary vector defining a direction of propagation of wave).

As a consequence, after back-substituting (16) into (4), we get the matrix problem

$$
\left[\begin{array}{ll}
K_{F F} & K_{F V} \\
K_{V F} & K_{V V}
\end{array}\right] \cdot\left[\begin{array}{l}
\lambda \\
A
\end{array}\right]=\left[\begin{array}{l}
L_{F} \\
L_{V}
\end{array}\right]
$$

where $K_{F F}$ and $K_{V V}$ are the matrix blocks of pure FEM part and pure VTCR part, respectively. $K_{F V}$ and $K_{V F}$ denote the matrix blocks of couple terms of FEM and VTCR. $\lambda$ and $A$ are the unknown degrees of freedom to be found. The terms of $K_{F F}$ are calculated by the Gauss integration. The $K_{V V}, K_{F V}$ and $K_{V F}$ terms are calculated through analytical calculations. The exponential form of the VTCR shape functions allow one to do this, at least on straight line boundaries. On the seldom cases where the boundaries are not straight, these terms are computed through numerical computations.

\section{Numerical Examples}

\subsection{Homogeneous Helmholtz problem with variation of scales in the solution}

The domain being considered is the square $\Omega=[0 \mathrm{~m} ; 0.5 \mathrm{~m}] \times[0 \mathrm{~m} ; 0.5 \mathrm{~m}]$ The prescribed boundary conditions are such that the exact solution is $u_{\text {exact }}=\sum_{j=1}^{5} \exp \left(i k(1+i \zeta)^{-1 / 2}(x\right.$. $\left.\left.\cos \left(\theta_{j}\right)+y \cdot \sin \left(\theta_{j}\right)\right)\right)$ with $\theta_{1}=5.6^{\circ}, \theta_{2}=12.8^{\circ}, \theta_{3}=18^{\circ}, \theta_{4}=33.5^{\circ}, \theta_{5}=41.2^{\circ}$ and 


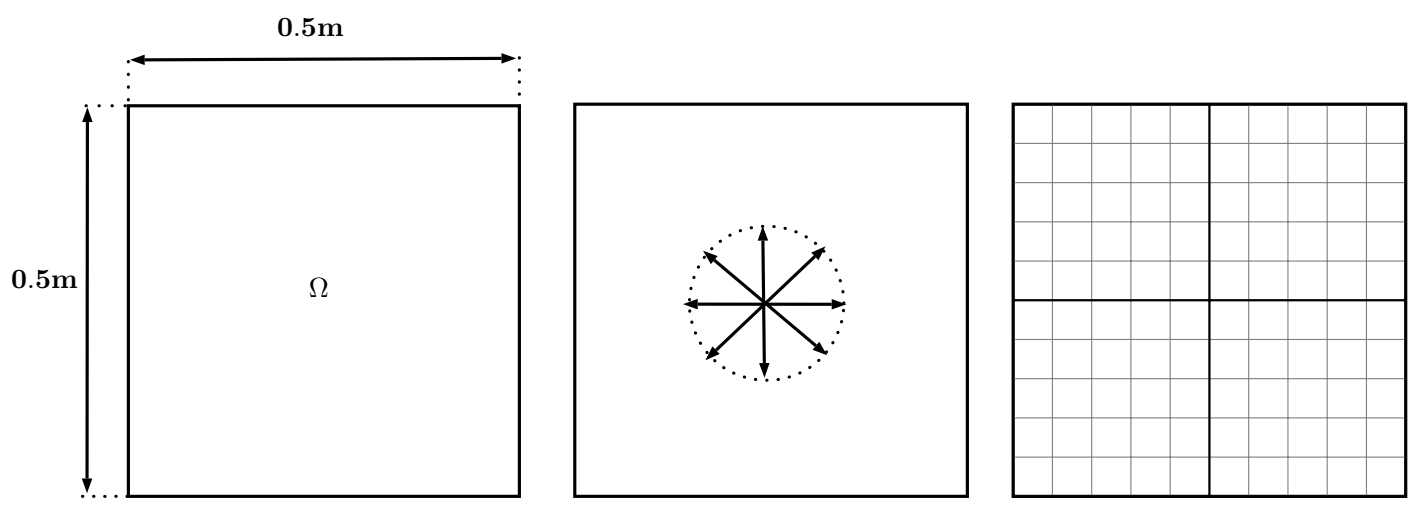

Fig. 2. Example considered in Sec. 3.1. Left: Definition of domain. Middle: VTCR wave directions discretization. Right: FEM mesh refinement.

$\zeta=0.0001$. We satisfy Eq. (1) with $k$ which varies from $5 \mathrm{~m}^{-1}$ to $72 \mathrm{~m}^{-1}$. This example is interesting, because it covers different scales (from slow varying scale with $k=5 \mathrm{~m}^{-1}$ to fast varying scale with $k=72 \mathrm{~m}^{-1}$ ) for the same model of problem, in the solution. It can be seen from this example how the WTDG method works from lower frequency problem to medium frequency problem. This definition of the problem and the discretization strategy can be seen in Fig. 2.

The WTDG strategy is used to find an approximation of $u_{\text {exact }}$. The WTDG formulation (4) is applied with Dirichlet boundary condition with $\alpha=0.0001$. For the FEM part, we use a regular squared mesh with a polynomial discretization of degree 1 . The discretization uses 10 elements per wave length. For the VTCR, we use a 2D regular polar repartition of the directions of the waves in $\Omega$. The discretization strategy complies with a geometrical heuristic criterion: $N_{e}=\delta k R_{e} /(2 \pi)$, where $N_{e}$ is the number of directions of waves, $\delta$ a parameter to be chosen ${ }^{24}$ and $R_{e}$ is the characteristic radius of $\Omega$. Here we take $\delta=10$. The convergence of the WTDG strategy is assessed by computing the relative error $\left|u_{\text {exact }}-u\right| /\left|u_{\text {exact }}\right|=\sqrt{\int_{\Omega}\left|u_{\text {exact }}-u\right|^{2} d \Omega / \int_{\Omega}\left|u_{\text {exact }}\right|^{2} d \Omega}$. We compare the pure FEM approach (which uses only a polynomial description), the pure VTCR approach (which uses only a wave description) and the WTDG approach (which uses at the same time the polynomial and the wave descriptions). Each time, the comparisons are done with a WTDG which is based on the same discretization than the pure FEM or the pure VTCR. The convergence curve is represented in Fig. 3.

As one can see, the WTDG presents a better behavior than the pure FEM or the pure VTCR. The pure FEM suffers from a lack of accuracy when the frequency becomes to be too high. The pure VTCR is not so efficient in the low frequency domain. This shows the benefits of using the WTDG method for finding the solution for low and medium frequency problems with the same descriptions, at the same time.

The convergence of the WTDG method depends on discretization strategies of both polynomial and wave shape functions. A study has been made to see how the discretization strategy of wave shape functions affects the performance of the WTDG method. With the 


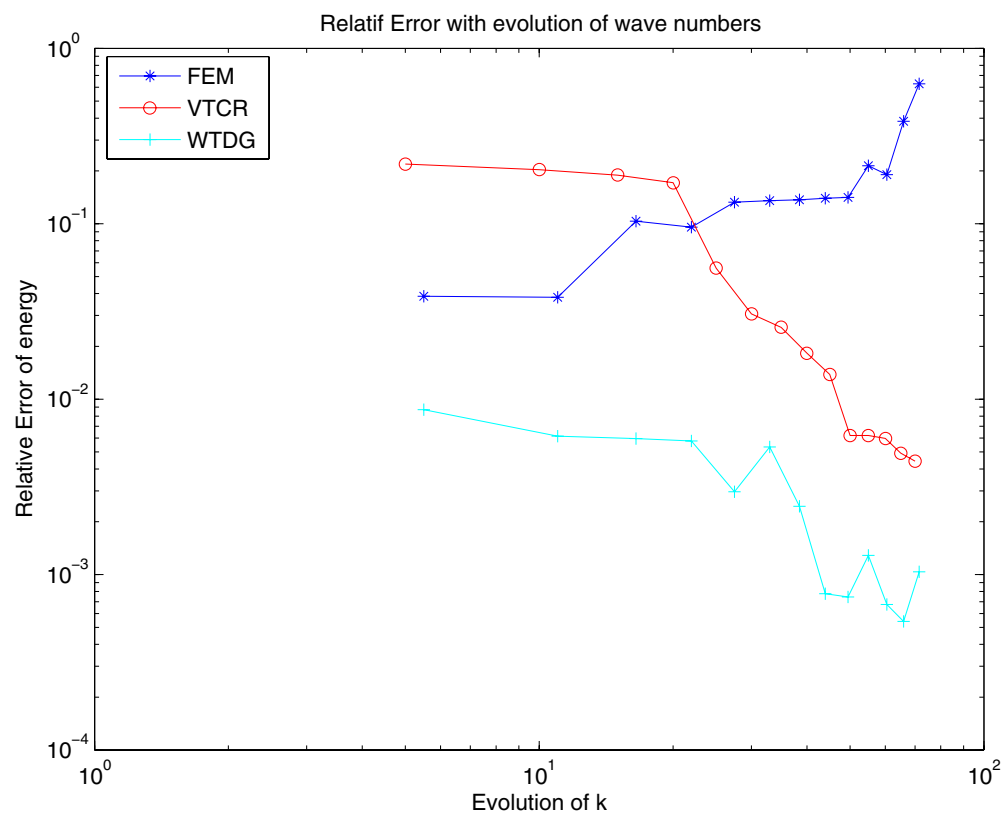

Fig. 3. The convergence curves for the example of Sec. 3.1. The FEM curve corresponds to the solution obtained with a pure FEM discretization explained in Sec. 3.1. The VTCR curve corresponds to the solution obtained with a pure VTCR discretization explained in Sec. 3.1. The WTDG curve corresponds to the solution obtained with an enrichment of the FEM shape functions with waves, according to the WTDG strategy explained in Sec. 2.4.

model of the same computational domain and the same boundary condition defined in this section, we take $k=25 \mathrm{~m}^{-1}$. Seven different discretization strategies of wave shape functions have been used to draw the convergence curves of the WTDG method as Fig. 4 shows. For each discretization strategy of wave functions, increasing of degree of freedoms of polynomial functions leads to convergence of the WTDG method. As one can see, for a fixed number of waves, the curves converge when the number of polynomial shape functions is increased. It can be seen that when using the same degree of freedoms of polynomial functions, enrichment of wave functions used in WTDG leads to more precise result. For the VTCR method, 32 wave shape functions are sufficient to have a precise simulation result in this problem. So here the convergence curve of FEM with 32 VTCR waves locates always in a zone of good precision.

\subsection{Nonhomogeneous Helmholtz problem with two scales in the solution}

We now consider the Helmholtz problem defined on $\Omega=[-0.5 \mathrm{~m} ; 0.5 \mathrm{~m}] \times[-0.5 \mathrm{~m} ; 0.5 \mathrm{~m}]$, with $k=100 \mathrm{~m}^{-1}, \theta_{1}=30^{\circ}, \theta_{2}=82^{\circ}, r_{d}=\left(k^{2}-k_{e}^{2}\right) \exp \left(i k_{e}(1+i \zeta)^{-1 / 2}\left(\cos \theta_{2} \cdot x+\right.\right.$ $\left.\left.\sin \theta_{2} \cdot y\right)\right), k_{e}=10 \mathrm{~m}^{-1}$ and $\zeta=0.0001$. We prescribe the boundary conditions such that the exact solution is $u_{\text {exact }}=\exp \left(i k(1+i \zeta)^{-1 / 2}\left(\cos \theta_{1} \cdot x+\sin \theta_{1} \cdot y\right)\right)+\exp \left(i k_{e}(1+\right.$ $\left.i \zeta)^{-1 / 2}\left(\cos \theta_{2} \cdot x+\sin \theta_{2} \cdot y\right)\right)$. This example is again interesting, because it corresponds to a nonhomogeneous Helmholtz problem with two scales in the solution (slow varying 


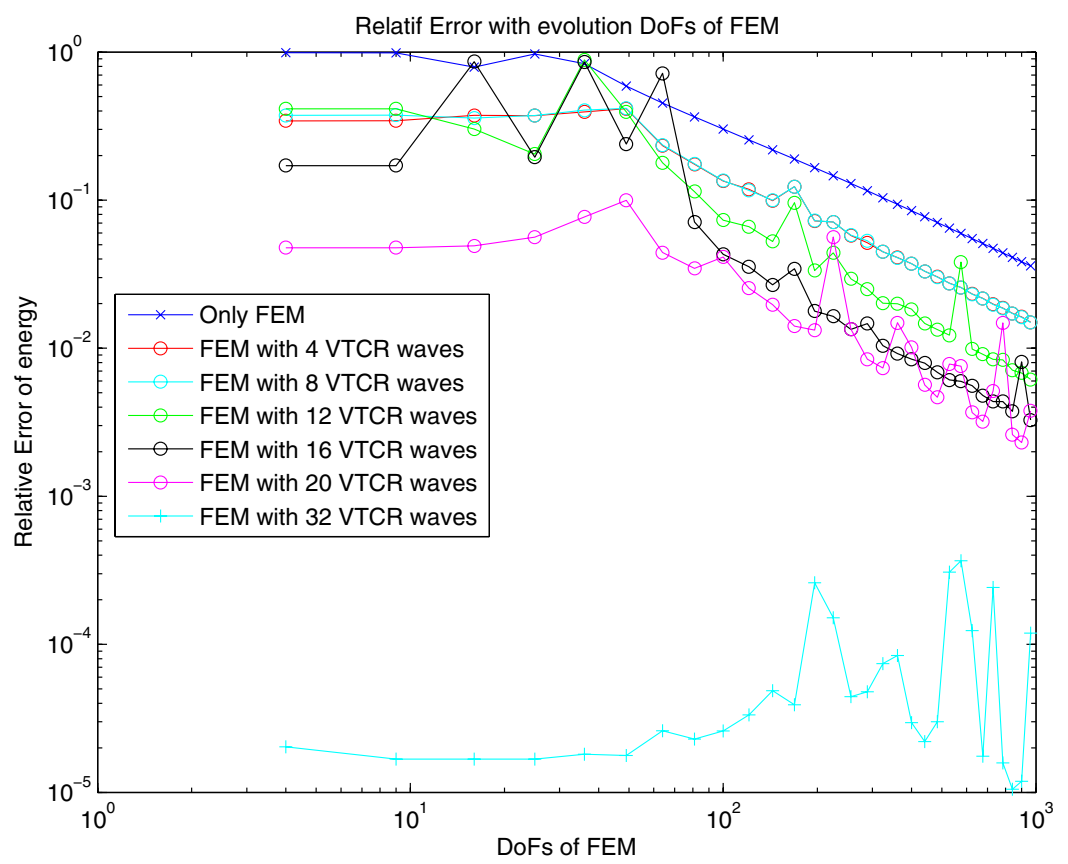

Fig. 4. The convergence curves for the example of Sec. 3.1. For each convergence curve, a fixed number of wave directions of VTCR part is chosen in WTDG strategy. The degree of freedoms of FEM part is varied in order to attain the convergence.

scale with $k_{e}$ and fast varying scale with $k$ ). The exact solution $u_{\text {exact }}$ is represented in Fig. 5.

We here consider the WTDG method, used with Dirichlet boundary condition with $\alpha=0.0001$. We mix the polynomial and wave shape functions. As we expect here that the polynomial shape functions help us to find the slow varying scale, and that the waves recover the fast varying scale, we consider a regular squared mesh with a polynomial discretization of degree 2 , and 2 waves, propagating in the $30^{\circ}$ and $210^{\circ}$ directions. The FEM descriptions uses 10 elements per wave length to simulate the slow varying scale. The exact solution of fast varying scale is taken directly as shape function of VTCR part. There is no need to add more shape function to simulate the fast varying scale. With such a choice, the solution given by the wave description, denoted $u_{\mathrm{VTCR}}$ and the polynomial description, denoted $u_{\text {FEM }}$ is depicted in Fig. 5 .

The comparison between the exact and the WTDG solution is represented in Fig. 6. According to the definition of the error in Sec. 3.1, the error is here $4.48 \times 10^{-5}$. As one can see, the WTDG method is able to recover the exact approximation with a very good accuracy and adopts the scale of the approximation to the physics of the problem.

\subsection{The WTDG method applied with different types of approximations}

We finally consider the computational domain defined in Fig. 7. This $L$ shape domain is filled with a fluid with $k=30 \mathrm{~m}^{-1}$ and $\zeta=0.0001$. The boundary conditions are such 

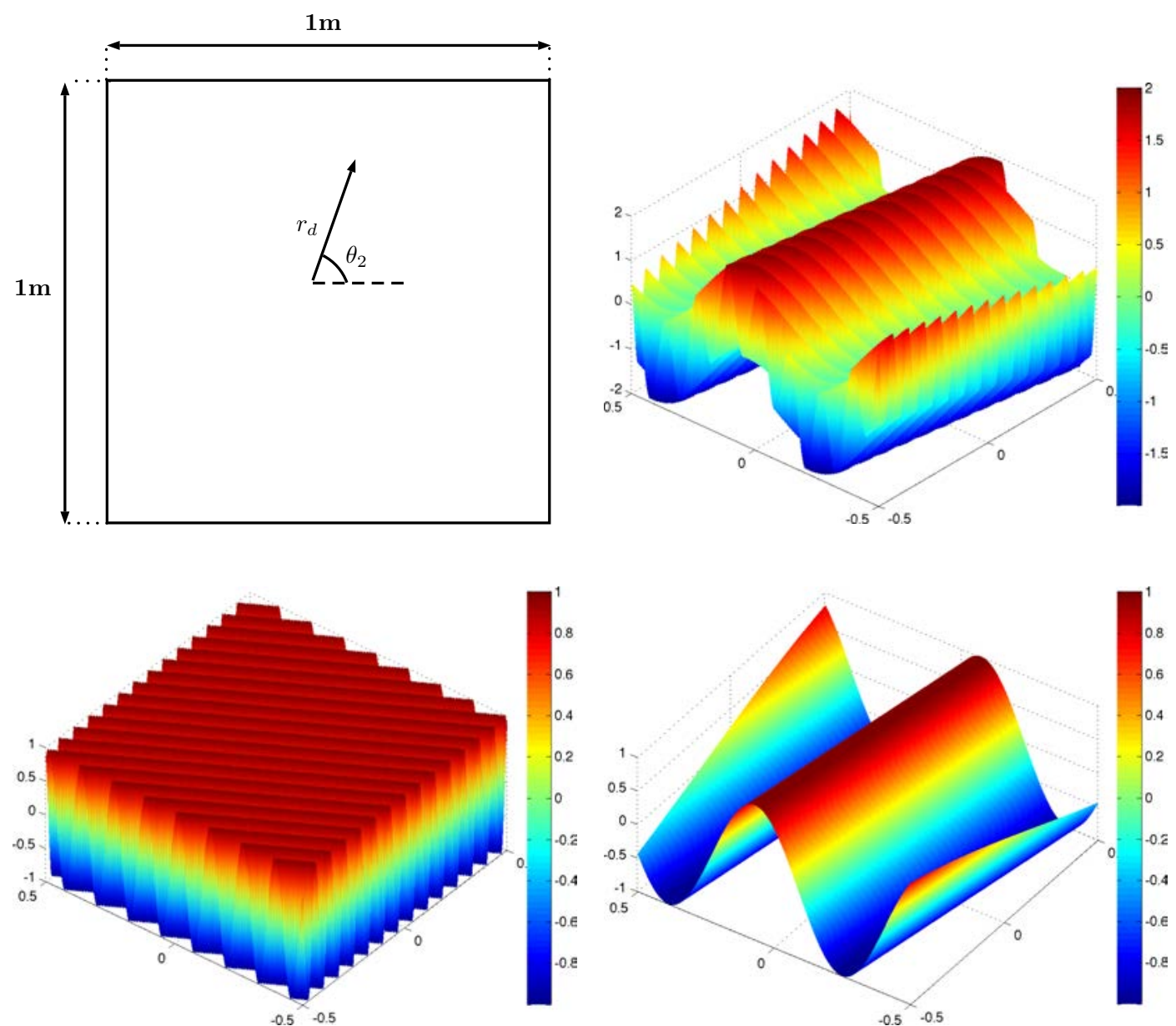

Fig. 5. Example considered in Sec. 3.2. Up left: Definition of the computational domain. Up right: Exact solution $u_{\text {exact }}$. Down left: Representation of the fast varying scale result simulated by VTCR part $u_{\mathrm{VTCR}}$. Down right: Representation of the slow varying scale result simulated by FEM part $u_{\mathrm{FEM}}$.
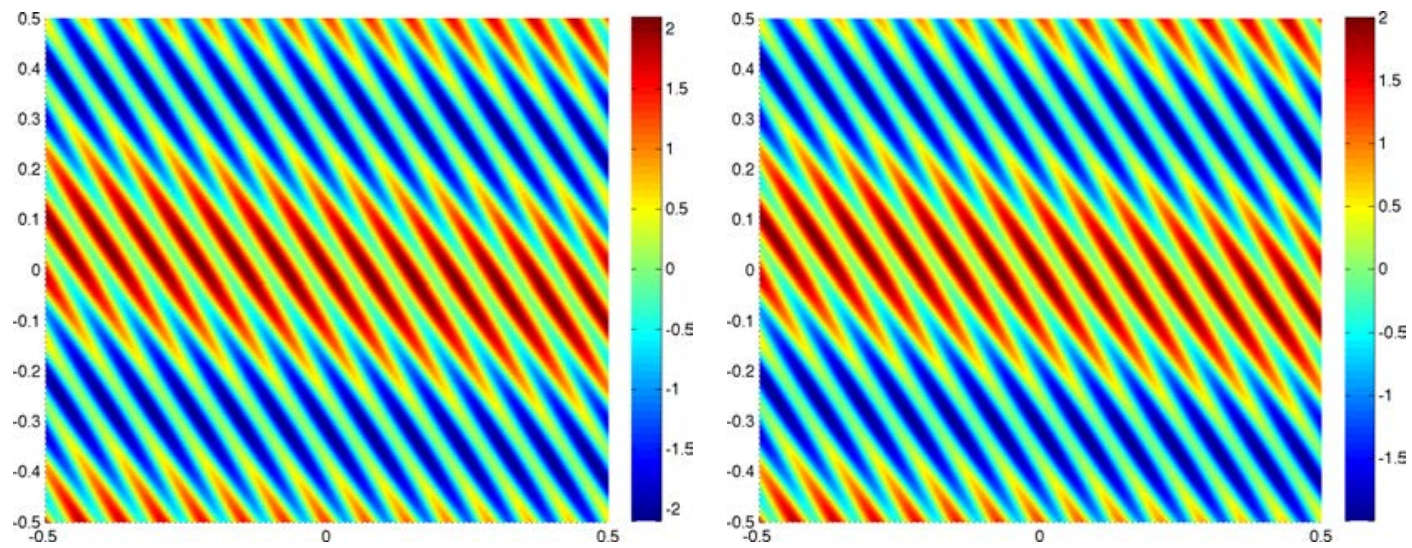

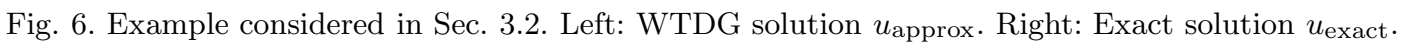



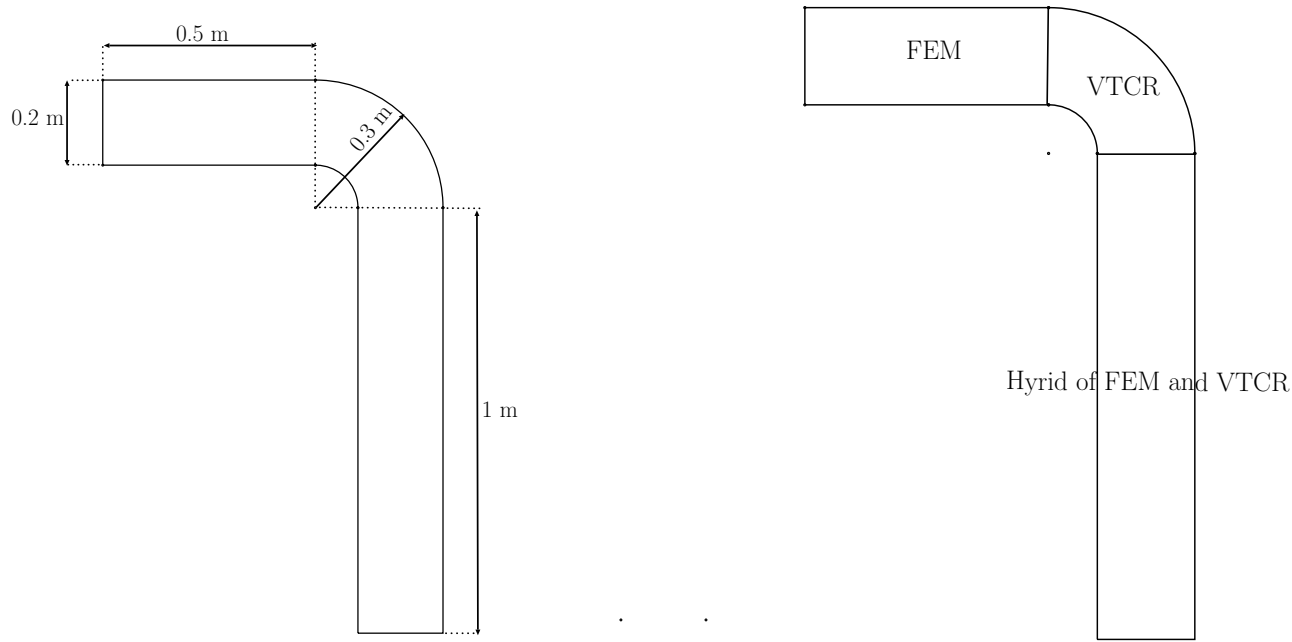

Fig. 7. Example considered in Sec. 3.3. Left: Computational domain $\Omega$. Right: Selected discretizations in the subdomains.

that the exact solution is $u_{\text {exact }}=\exp \left(k(1+i \zeta)^{-1 / 2} \cdot \cos \left(\frac{\pi}{3}\right) x+k(1+i \zeta)^{-1 / 2} \cdot \sin \left(\frac{\pi}{3}\right) y\right)+$ $\exp \left(k(1+i \zeta)^{-1 / 2} \cdot \sin \left(\frac{\pi}{3}\right) x+k(1+i \zeta)^{-1 / 2} \cdot \cos \left(\frac{\pi}{3}\right) y\right)$.

On this example, we use different kinds of approximations: even a pure FEM approximation, or a pure VTCR approximation, or a mix between polynomial and wave shape functions (see Fig. 7). The formulation of Discontinuous Galerkin method allows this possibility. This example differs from the one given in Ref. 25 by the fact that here, in one subdomain of $\Omega$, we mix the approximations. In order to be sure to have a good approximation, we select 20
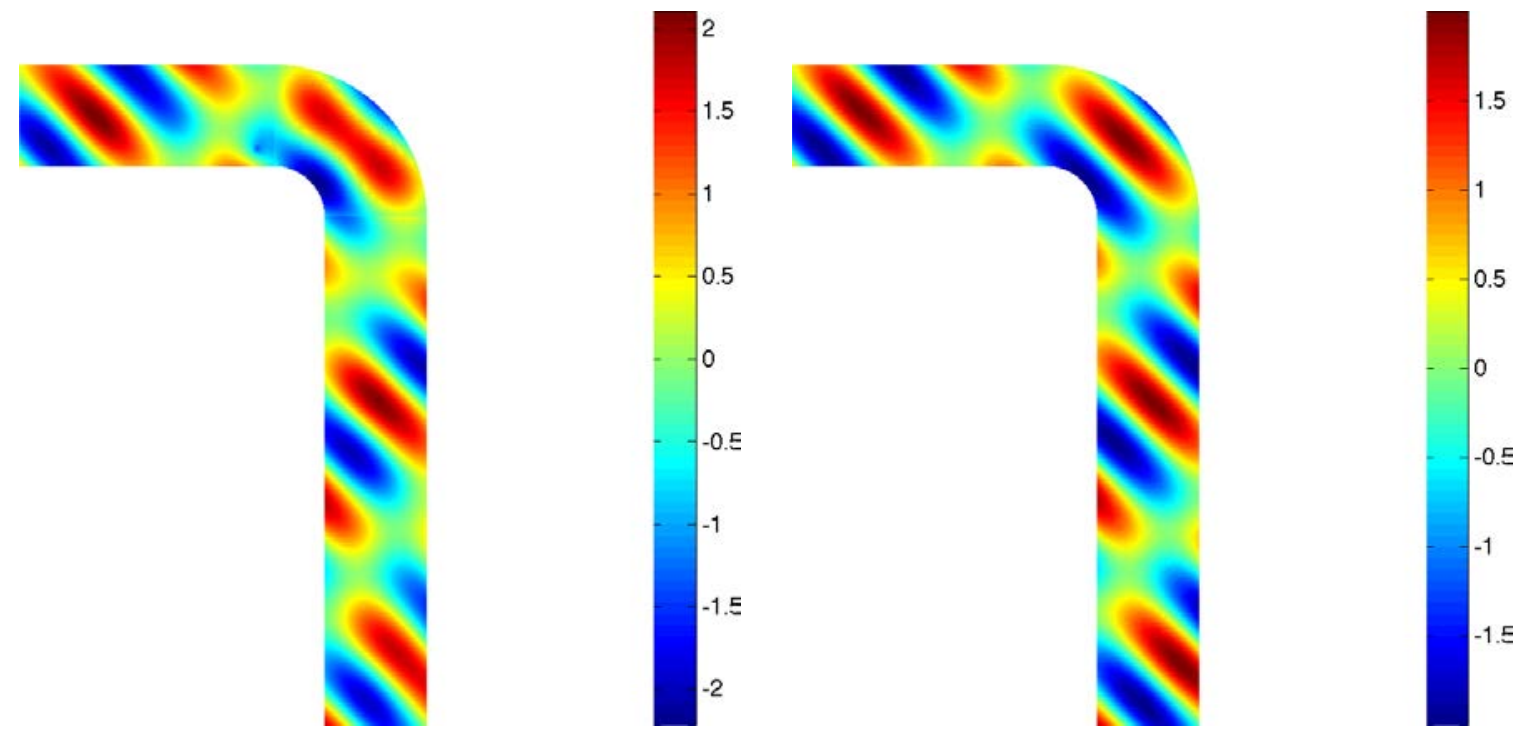

Fig. 8. Example considered in Sec. 3.3. Left: WTDG solution. Right: Exact solution. 
elements of degree 1 per wave length for the FEM description, and $\delta=14$ for the VTCR description. The WTDG uses $\delta=17$ for the wave approximations and 6 elements of degree 1 in the subdomain. Again, Dirichlet boundary conditions with $\alpha=0.0001$ allow us to prescribe $u_{\text {exact }}$ on the boundaries of $\Omega$.

The exact solution and the WTDG solution are depicted in Fig. 8. As one can see, the solutions are very closed. This is because the formulation of weak Trefftz Galerkin method allows the couple using FEM and VTCR and their hybrid. According to the definition of the error in Sec. 3.1, the error is here $2.187 \times 10^{-2}$. It can be deduced from this example that all combinations of methods such as pure FEM, pure VTCR, hybrid of FEM and VTCR can be integrated together in one complex geometry problem. In each subdomain the concrete method can be chosen depend on specific requirement of engineering problems.

\section{Conclusion}

This paper proposed an hybrid use of FEM and VTCR thanks to the Weak Trefftz Discontinuous Galerkin method. It is illustrated on the Helmholtz problem. The WTDG method allows one to use a combination of traditional FEM shape functions and VTCR shape functions. It is based on a variational formulation which is equivalent to the reference problem. All the conditions such as the governing equation, the transmission continuity and the boundary conditions are included in the formulation. No a priori constraint is needed for the definition of the shape functions that are used. As a consequence, any shape function can be used, with no difficulty. It gives then to the WTDG method a great flexibility, as the used can select polynomials or wave shape functions (or a combination of them) very easily in the approximated space, with no restriction. It is successfully illustrated on different examples of different complexity, homogeneous or not, with sometimes two scales in the solution. An extension to vibro-acoustic problems would present no particular difficulty and will be addressed in future works.

\section{References}

1. O. C. Zienkiewicz et al., The Finite Element Method (McGraw-hill, London, 1977).

2. A. Deraemaeker, I. Babuska and P. Bouillard, Dispersion and pollution of the FEM solution for the Helmholtz equation in one, two and three dimensions, Int. J. Numer. Methods. Eng. 46 (1999) 471-499.

3. E. Trefftz, Ein gegenstück zum ritzschen verfahren, in Second International Congress on Applied Mechanics (1926), pp. 131-137.

4. T. Strouboulis and R. Hidajat, Partition of unity method for Helmholtz equation: $q$-convergence for plane-wave and wave-band local bases, Appl. Math. 51 (2006) 181-204.

5. O. Cessenat and B. Despres, Application of an ultra weak variational formulation of elliptic PDEs to the two-dimensional Helmholtz problem, SIAM J. Numer. Anal. 35 (1998) $255-299$.

6. T. Huttunen, J. Kaipio and P. Monk, An ultra-weak method for acoustic fluid-solid interaction, J. Comput. Appl. Math. 213 (2008) 166-185.

7. P. Monk and D. Wang, A least-squares method for the Helmholtz equation, Comput. Methods Appl. Mech. Eng. 175 (1999) 121-136. 
8. G. Gabard, P. Gamallo and T. Huttunen, A comparison of wave-based discontinuous Galerkin, ultra-weak and least-square methods for wave problems, Int. J. Numer. Methods Eng. 85 (2011) 380-402.

9. C. J. Gittelson, R. Hiptmair and I. Perugia, Plane wave discontinuous Galerkin methods: Analysis of the $h$-version, ESAIM: Math. Model. Numer. Anal. 43 (2009) 297-331.

10. G. Fairweather and A. Karageorghis, The method of fundamental solutions for elliptic boundary value problems, Adv. Comp. Math. 9 (1998) 69-95.

11. A. Barnett and T. Betcke, Stability and convergence of the method of fundamental solutions for Helmholtz problems on analytic domains, J. Comput. Phys. 227 (2008) 7003-7026.

12. C. Farhat, I. Harari and L. Franca, The discontinuous enrichment method, Comput. Methods Appl. Mech. Eng. 190 (2001) 6455-6479.

13. C. Farhat, R. Tezaur and J. Toivanen, A domain decomposition method for discontinuous Galerkin discretizations of Helmholtz problems with plane waves and Lagrange multipliers, Int. J. Numer. Methods Eng. 78 (2009) 1513-1531.

14. P. Bouillard and S. Suleaub, Element-free Galerkin solutions for Helmholtz problems: Fomulation and numerical assessment of the pollution effect, Comput. Methods Appl. Mech. Eng. 162 (1998) 317-335.

15. E. Perrey-Debain, J. Trevelyan and P. Bettess, Wave boundary elements: A theoretical overview presenting applications in scattering of short waves, Eng. Anal. Bound. Elem. 28 (2004) 131-141.

16. H. Bériot, E. Perrey-Debain, M. Ben Tahar and C. Vayssade, Plane wave basis in Galerkin bem for bidimensional wave scattering, Eng. Anal. Bound. Elem. 34 (2010) 130-143.

17. W. Desmet, P. Sas and D. Vandepitte, An indirect Trefftz method for the steady-state dynamic analysis of coupled vibro-acoustic systems, Comput. Assisted Mech. Eng. Sci. 8 (2001) 271-288.

18. B. Van Genechten, O. Atak, B. Bergen, E. Deckers, S. Jonckheere, J. S. Lee, A. Maressa, K. Vergote, B. Pluymers and D. Vandepitte, An efficient wave based method for solving Helmholtz problems in three-dimensional bounded domains, Eng. Anal. Bound. Elem. 36 (2012) 63-75.

19. P. Ladevèze, A new computational approach for structure vibrations in the medium frequency range, C. R. Acad. Sci. Paris 332 (1996) 849-856.

20. P. Rouch and P. Ladevèze, The variational theory of complex rays: A predictive tool for mediumfrequency vibrations, Comput. Methods Appl. Mech. Eng. 192 (2003) 3301-3315.

21. P. Ladevèze, L. Blanc, P. Rouch and C. Blanzé, A multiscale computational method for mediumfrequency vibrations of assemblies of heterogeneous plates, Comput. Struct. 81 (2003) 12671276.

22. H. Riou, P. Ladevèze and P. Rouch, Extension of the variational theory of complex rays to shells for medium-frequency vibrations, Sound Vib. 272 (2004) 341-360.

23. H. Riou, P. Ladevèze and B. Sourcis, The multiscale VTCR approach applied to acoustics problems, Comput. Acoust. 16 (2008) 487-505.

24. L. Kovalevsky, H. Riou and P. Ladevèze, On the use of the variational theory of complex rays for the analysis of 2-D exterior Helmholtz problem in an unbounded domain, J. Wave Motion 50 (2013) 428-436.

25. P. Ladevèze and H. Riou, On Trefftz and weak Trefftz discontinuous Galerkin approaches for medium-frequency acoustics, Comput. Methods Appli. Mech. Eng. 278 (2014) 729-743. 